\title{
Toma de Decisiones en Nodos del Sistema Distribuido TLÖN
}

\section{Decision Making in Distributed System TLÖN Nodes}

\author{
John Edwar González ${ }^{1}$, Jorge Eduardo Ortiz ${ }^{2}$ Y Henry Zárate
}

\section{RESUMEN}

El primer paso hacia la Social-inspiración en un sistema distribuido que opere sobre una red Ad Hoc, es implementar la característica de racionalidad en nodos que deseen unirse al sistema, de manera que puedan decidir sobre ingresar o no, teniendo en cuenta los recursos que éste les ofrece. En este artículo se expone las diferentes etapas del proceso para el ingreso del nodo a la red, los diferentes métodos de toma de decisiones en cada etapa y la justificación de la elección de cada uno de ellos como los que más se ajustan dado los parámetros que se manejan en este tipo de redes y el paradigma de Social-inspiración que se está aplicando.

Palabras Clave: Negociación; Recursos; Red ad hoc; Social-Inspiración.

\section{ABSTRACT}

The first step towards Social-inspiration in a distributed system that operates on an Ad Hoc network is to implement the rationality characteristic in nodes that want to be part of it, so that they can decide to enter or not, taking into account the resources that this offers. This article presents the different stages of the process for the entry of the node to the network, the different methods of decision making in each stage and the justification of the choice of each one of them as the ones that fit the most given the parameters that are managed in this type of networks and the Social-inspiration paradigm that is being applied.

Keywords: Negotiation; Resources; Ad hoc Network; Social-Inspiration.

\section{INTRODUCCIÓN}

En las últimas décadas, la ciencia ha estado muy interesada en comprender los procesos biológicos que hacen posible la vida, estos procesos están caracterizados por su complejidad y perfección; es por esto que los científicos han querido fundarse en estos métodos para aplicarlos a problemas de la ingeniería (Maldonado \& Gomez, 2011). El éxito de paradigmas bioinspirados se observa por ejemplo en redes neuronales, lógica difusa, o en inteligencia artificial.
De igual manera, se ha tenido mucho interés en formular matemáticamente diferentes conceptos sociales, como por ejemplo la justicia y la teoría de la elección social (Gaertner, 2009) como base para una equitativa distribución de recursos entre los miembros de sistemas distribuidos como se observa en (Pitt et al., 2015), la social-inspiración, al igual que la bioinspiración, intenta aplicar todos esos conceptos sociales, y ha tomado fuerza a la hora de solucionar problemas de ingeniería teniendo como punto de partida el éxito de las comunidades.

\footnotetext{
${ }^{1}$ Ingeniero Electrónico. Investigador, Universidad Nacional de Colombia, Cra. 45, Bogotá, joegonzalezor@unal.edu.co.

2 PhD. en Ingeniería de Sistemas y Computación. Profesor Asociado, Ingeniería de Sistemas e Industrial, Universidad Nacional de Colombia, Cra. 45, Bogotá, jeortizt@unal.edu.co.

${ }^{3}$ Magister en Telecomunicaciones. Investigador Colciencias, Universidad Nacional de Colombia, Cra. 45, Bogotá, hzaratec@unal.edu.co.
} 
En el caso de los enjambres de abejas y colonias de hormigas, el estar acompañados por miembros de

la misma especie, y su disposición a la hora de cumplir las tareas que les corresponden dependiendo del rango de su organización, ha determinado su supervivencia en la naturaleza. En el caso de los seres humanos, este hecho es similar, pero aquí el concepto tiene un sentido más amplio como lo expone (Rawls, 2012) en donde la sociedad se define como una asociación de personas que conforman una empresa cooperativa y que se ayudan entre sí para obtener el bienestar de todos; y tienen como base la justicia y la democracia. Con base en esto, se ha propuesto en el grupo de investigación TLÖN de la Universidad Nacional de Colombia, la implementación del paradigma de la social-inspiración en sistemas distribuidos que se implementen sobre redes Ad Hoc de manera que los nodos se comporten como personas al querer ingresar a una organización que les brinde recursos de manera justa y en donde puedan satisfacer requerimientos que por limitaciones propias del dispositivo no puedan cumplir. En este proyecto en específico, se va a abordar un primer paso que conllevaría a alcanzar ese proyecto general y es aplicar la capacidad de toma de decisiones a los nodos de un sistema distribuido, de manera que ellos puedan decidir, con base en los recursos que le ofrece la red, si desean ingresar o no.

Inicialmente se dará el concepto de sistema distribuido, enseguida se introducirá el término de toma de decisiones y se definirá el método multicriterio Scoring que es el utilizado por parte del nodo como el primer paso para la decisión de ingreso a la red, luego se define la teoría de la negociación que es lo que el nodo y la red utilizan para ponerse de acuerdo sobre la cantidad de recursos que el nodo aporta a la red para su ingreso definitivo.

Finalmente se muestra la aplicación de los conceptos en el modelo de aplicación del sistema distribuido TLÖN, en específico las interacciones entre dos nodos solitarios (es decir, que no pertenecen a ninguna red Ad Hoc) y entre un nodo solitario y la red, a la hora de la conformación del sistema distribuido.

\subsection{Sistema Distribuido y Red Ad Hoc}

Un sistema distribuido (Fitzek \& Katz, 2014) se puede definir como una colección de nodos que están separados físicamente, pero que se unen entre sí por algún medio de telecomunicaciones; cada nodo tiene características propias, sus recursos son heterogéneos. Un sistema distribuido se puede implementar sobre cualquier red de comunicaciones y permite la comunicación entre múltiples nodos; en él, el usuario accede a cualquier componente de cualquier nodo, y lo percibe como si se tratara de un recurso local y en donde todos los dispositivos forman un solo equipo de cómputo.

En el proyecto que se va a diseñar en el grupo de investigación, se tendrá una red Ad Hoc como base para la construcción del sistema distribuido. Las redes $\mathrm{Ad} \mathrm{Hoc}$ son una colección de dos o más dispositivos Inalámbricos que poseen la capacidad de comunicarse entre ellos y tienen como principal característica la no utilización de infraestructura física fija ni administración centralizada (Loo et al., 2012) sino que basan su funcionamiento en la interacción de sus nodos quienes se encargan de compartir sus recursos para el uso de toda la red. Cada nodo puede ser un dispositivo como un celular, un computador, una Tablet, entre otros, como se observa en la figura 1 ; los cuales poseen características y recursos heterogéneos.

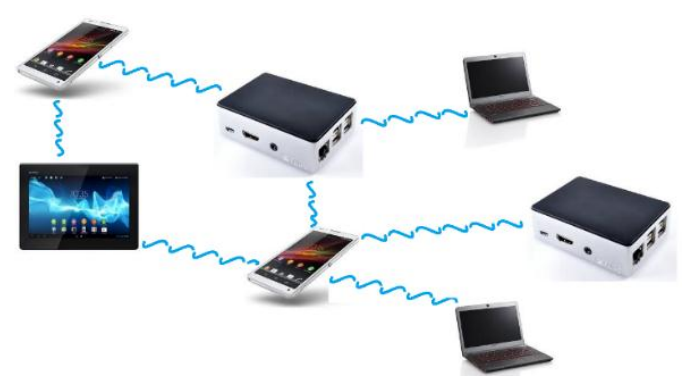

Figura 1. Imagen de una red ad hoc.

En particular, el procedimiento de unión de un nodo a una red Ad Hoc y a un sistema distribuido depende del protocolo de enrutamiento que se use, pero usualmente lo que ocurre es que el nodo se comunica con el nodo más cercano de la red y no hay un medio de registro en el sistema y mucho menos se tiene en cuenta qué recursos le está ofreciendo la red, de manera que el nodo puede salir perjudicado en cuanto a cantidad de recursos si ingresa a una red que no llene sus expectativas y que por el contrario sólo le solicite recursos. O por otro lado, la red puede estar aceptando un nodo que es egoísta y que solo va a explotar recursos de la red y en realidad no va a 
aportar nada, de allí que el proyecto esté enfocado a que el nodo pueda tomar la decisión de ingresar dependiendo de lo que la red le ofrezca y ésta a la vez pueda admitir o denegar la entrada a un nodo de acuerdo a su comportamiento.

En este ámbito, se podría tener un escenario muy acorde al incluir la capacidad de toma de decisiones en los nodos, de manera que autónomamente eligieran la posibilidad de ingresar a un sistema distribuido, dependiendo de lo que éste le ofrezca. Como se mencionó anteriormente este sería un primer paso hacia la social-inspiración y de paso haría que la red conservara la principal característica de las redes Ad Hoc que es la auto-organización, ya que el nodo autónomamente sin necesidad de intervención del usuario, evaluaría los recursos que le está ofreciendo el sistema distribuido y con base en los que él tiene, elegiría si ingresa o no al sistema.

\subsection{Toma de Decisiones}

La toma de decisiones es el proceso mediante el cual, se realiza la elección de una opción entre varias, mediante un método que pueda resaltar algunas de las opciones de otras, de manera que se puedan organizar jerárquicamente (Saaty, 1990).

A la hora de abarcar la teoría de la toma de decisiones, esta se divide en tres grandes bloques de análisis (Vitoriano, 2007), así:

- La teoría de decisión con incertidumbre o riesgo: En este caso, la toma de decisiones se realiza teniendo en cuenta que las consecuencias no pueden ser controladas, ya sea porque no se tiene suficiente información o porque las mismas están sujetas al azar.

- Decisión multicriterio: Aunque se sabe las consecuencias de escoger una opción, no se sabe qué es lo mejor entre todas ellas, algunos de sus objetivos pueden estar en conflicto.

- Teoría de Juegos: Las consecuencias de la decisión no dependen de la decisión adoptada, sino también de la que elijan otros jugadores.

En la teoría de la elección es necesario tener en cuenta la perspectiva desde donde se está tomando la decisión, en los casos de las redes Ad Hoc, no sólo es necesario tener en cuenta la toma de decisiones desde el punto de vista del nodo, sino también, desde el punto de vista de la red, como una articulación de las opiniones de los miembros que componen esa comunidad, como en una democracia, en donde las acciones públicas están determinadas por los miembros de la sociedad; estas opiniones son agregadas a una preferencia social que refleja la opinión general de la sociedad (Gaertner, 2009).

\subsection{Negociación Cooperativa.}

El problema aquí se refiere a la elección de una alternativa cuando hay varios actores que están en conflicto de intereses; pero todo se desarrolla en un marco de cooperación. Al final es un problema de consenso, en donde la decisión final solo puede ser tomada cuando las partes en disputa están de acuerdo; existe la posibilidad de que haya una amenaza cuando ambas partes fracasan en alcanzar un acuerdo. Por lo cual las decisiones tienen que ser tomadas teniendo en cuenta el punto en disputa.

En la teoría de negociación lo más importante son las combinaciones de la utilidad de las partes en conflicto, en este caso, el nodo y la red. En la solución de negociación de Nash aplicada en (Gaertner, 2009) (Ozdaglar, 2010), se observa lo que pasa cuando ambas partes actúan en forma racional, maximizando la utilidad de ambas partes, incluso cuando el resultado de la salida no es justo.

Formalización: Sea $\mathrm{S}$ un conjunto de vectores de utilidad, los puntos $u_{1}^{*}$ y $u_{2}^{*}$ las utilidades de los jugadores si estos no se ponen de acuerdo y la salida negociada como puntos $\bar{u}$ y $\bar{v}$. Entonces el objetivo sería encontrar una función (1) la cual realice el mapeo de los posibles puntos de utilidad de no acuerdo y los puntos de vectores de utilidad hacia los puntos de la salida negociada, así:

$$
(\bar{u}, \bar{v})=f\left(S, u^{*}, v^{*}\right)
$$

El problema ahora es encontrar esa función; para ello Nash definió una serie de axioma que debe cumplir la función con el fin de hacer una negociación justa, en esta situación en donde los individuos son racionales, conocen las preferencia de los otros y tienen la misma habilidad de negociación, la ecuación (2) muestra las características de esa función. 


$$
(\bar{u}, \bar{v})=\max _{(u, v) \in S}\left(u-u^{*}\right)\left(v-v^{*}\right)
$$

- Axioma 1. Invariancia con respecto a las transformaciones de la utilidad, es decir, si los actores de las negociaciones transforman sus escalas de utilidades de manera independiente, las coordenadas de la solución cambiarán en las mismas escalas de transformación la utilidad.

- Axioma 2. Debilidad de la eficiencia de Pareto, la salida negociada debe estar en la frontera de Pareto de la región de utilidades.

- Axioma 3. Simetría, si una persona no sabe distinguir entre su status quo y sus posibles distribución de utilidad, la solución tendrá que ser igual para todos los jugadores.

- Axioma 4. Independencia de las alternativas irrelevantes o consistencia de la contracción, la solución para $\mathrm{T}$ deba también ser la solución para $S$, si $T \subset S$.

En el principio de concesiones alternativas de Zeuthen que se detalla en (Crawford, 1980); Zeuthen propuso un procedimiento en el que las partes involucradas en la negociación hacen ofertas de forma alternada. Este método de negociación constituye una solución a la negociación de Nash e incluye elementos de la teoría de juegos no cooperativos. Luego, en (Kalai, 1977) en su solución de negociación, se propone el reemplazo del axioma 4 de independencia de Nash por un axioma de monotonicidad de manera que si hay una expansión en la región de utilidad y si para el jugador 1 el nivel de utilidad permanece constante, el nivel de utilidad del jugador 2 debe aumentar ligeramente.

\section{MATERIALES Y MÉTODOS}

El primer paso hacia la social-inspiración en el sistema distribuido TLÖN (Zarate Ceballos et al,. 2015) es brindarle al nodo la capacidad de racionalidad de manera que enfrente el dilema de pertenecer al sistema distribuido de manera similar a como una persona lo haría al tener que tomar la decisión sobre su ingreso a una organización social que cuenta con unos beneficios específicos.
Este sistema contaría con justicia, el cual es un principio orientador que lo distinguiría de otros sistemas distribuidos, en donde a los nodos que más aportan recursos en relación a sus recursos disponibles, se les asignan más recursos a la hora de necesitarlos, y viceversa, a los nodos que menos colaboren con recursos, de manera proporcional se les restringirá la ayuda a la hora que la necesiten.

Esto sería un gran incentivo ya que los nodos además del hecho de poder acceder a los recursos heterogéneos con los que cuenta una red Ad Hoc, podrían mejorar su reputación, el cual será un parámetro medible; además con éste podrán tener aún más posibilidades y prioridad a la hora de acceder a recursos del sistema.

Existen dos escenarios principales a saber en los cuales un nodo puede estar inmerso al tomar la decisión de acceder al sistema distribuido TLÖN:

- Unión de Nodo con otro Nodo Solitario. En este caso, el nodo se encuentra solo y se comportaría como un nodo Dios, es decir, el nodo sería el encargado de crear la red Ad Hoc y formar el sistema distribuido sobre ella; de ahí en adelante los demás nodos buscarían hacer parte de esa red.

- Nodo con una red ya constituida. Es el caso en donde el nodo se encuentra con otra red la cual ya es constituida por mínimo dos nodos.

Esta diferenciación se hace, ya que es diferente la decisión que realiza un nodo al querer formar una red Ad Hoc con otro nodo, al proceso de aceptación que tiene una red ante un nodo solitario, ya que ésta debe tener en cuenta a los participantes actuales de la red, pues es necesario tener cierta reserva a la hora de la admisión para evitar el hecho de que se acepte a un nodo egoísta que vaya a aprovecharse de los recursos actuales de la red y no aporte nada al sistema. Este efecto se puede observar en la teoría del nodo egoísta que se expone en (Toh et al., 2010).

\subsection{Nodo con Otro nodo Solitario}

En este escenario, una de las principales razones para la unión con otro nodo es ganar la reputación de haber sido uno de los nodos que creo la red Ad Hoc y 
el Sistema Distribuido que está sobre ella, y por ende una de las principales características que posee el nodo es el altruismo desde una perspectiva sociológica como se define en (Stiefken Arboleda, 2008), es decir, el hecho de dar de sí lo más posible, para aumentar su estatus e integración social, lo cual es muy apreciado en general en las redes Ad Hoc. Para el ingreso se realiza el proceso que se observa en la figura 2.

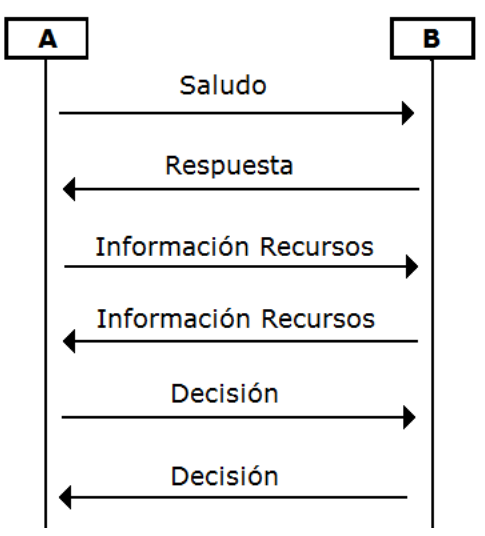

Figura 2. Proceso de formación de la red Ad Hoc entre dos nodos.

Para ello el nodo estará escaneando el espectro buscando la comunicación con nodos y/o redes Ad Hoc, en este caso, al encontrar un nodo, el primer paso que realiza es un saludo, como se observa en la figura 2, de A hacia $\mathrm{B}$, y el nodo $\mathrm{B}$ le envía una respuesta; una vez el nodo A recibe esa respuesta, envía la información de sus recursos, es decir, su capacidad de disco duro, Ram, procesamiento, disponibles y totales, además de sus periféricos como teclado, cámaras, micrófonos, etc., y el nodo $\mathrm{B}$ a su vez responde de manera similar con su propia información de recursos; en seguida, mediante un proceso de toma de decisiones multicriterio llamado Scoring cada nodo decide si crear o no la red.

\subsubsection{Método de Decisión Multicriterio Discreto Scoring.}

Como se mencionó anteriormente la toma de decisiones es el proceso mediante el cual, se realiza la elección de una opción entre varias, mediante un método que pueda resaltar algunas de las opciones de otras, de manera que se puedan organizar de manera jerárquica. La elección consiste en elegir lo mejor entre lo posible.
En los métodos de decisión multicriterio lo posible se refiere a la enumeración de todas las alternativas o el establecimiento de una región factible (Vitoriano, 2007). La región factible consiste en el conjunto de alternativas cuando estas no se pueden definir de manera precisa, este conjunto puede ser discreto o continuo; a su vez, la región factible puede ser limitada de manera estricta, mediante restricciones o de manera flexible por niveles de aspiración.

En cuanto a lo mejor, se debe elegir uno o más criterios para poder resaltar esa alternativa de las demás. Entre mayor número de criterios hayan, el problema de elección se vuelve un poco más complejo y se pueden resolver con métodos denominados de optimización multiobjetivo. Si lo posible viene definido por un conjunto discreto de alternativas existen métodos multicriterio discretos para resolver el problema, como el método multicriterio discreto Scoring.

Este método es una manera rápida y sencilla para realizar una elección en un problema de decisión; el proceso para realizar una decisión Multicriterio con este método es el siguiente:

- Identificar el propósito del problema. Este paso consiste en identificar qué es lo que se desea alcanzar.

- Identificar las alternativas. Son las opciones que podrían ayudar a la consecución del propósito que se trazó en el primer paso.

- Listar los criterios que van a ser tenidos en cuenta a elegir la mejor alternativa.

- Asignar una ponderación para cada uno de los criterios. es decir, indicar qué tan importante es para el decisor el criterio en cuestión mediante la siguiente escala de 1 a 5 , como se observa en la Tabla 1. 
Tabla 1. Ponderación criterios

\begin{tabular}{|c|c|}
\hline Escala & Significado \\
\hline 1 & Muy poco importante \\
\hline 2 & Poco importante \\
\hline 3 & Importancia media \\
\hline 4 & Algo importante \\
\hline 5 & Muy importante \\
\hline
\end{tabular}

- En la tabla 2, se observa la tabla de satisfacción de criterios, en este paso se indica, para cada alternativa; en cuanto se satisface cada uno de los criterios en una escala de 1 a 9 .

Tabla 2. Satisfacción de Criterios

\begin{tabular}{|c|c|}
\hline Escala & Significado \\
\hline 1 & Extra bajo \\
\hline 2 & Muy bajo \\
\hline 3 & Bajo \\
\hline 4 & Poco bajo \\
\hline 5 & Medio \\
\hline 6 & Medio alto \\
\hline 7 & Alto \\
\hline 8 & Muy alto \\
\hline 9 & Extra alto \\
\hline
\end{tabular}

- Calcular el Score para cada una de las alternativas, se puede hacer de la siguiente manera (3):

$$
S_{j}=\sum_{j} w_{i} r_{i j}
$$

Donde:

$r_{i j}=$ Valor de la alternativa $j$ para el criterio $i$, calculado en el paso 5 .

$w_{i}=$ Ponderación de cada criterio, calculado en el paso 4.

$S_{j}=$ Score para cada alternativa $j$

Finalmente se elige la alternativa que sea mayor.

Para el caso de toma de decisiones que se está manejando, se podría dejar de la siguiente manera.

- El propósito del problema es decidir si ingresar o no a la red Ad Hoc, y obtener mejores recursos o reputación en la red, esto se hace dependiendo de los recursos que le ofrezca la red comparándolos con los que el nodo posee.

- Las alternativas son: ingresar a la red o no hacerlo (Ingresar o No a la red).

- Criterios: Ram, procesador, disco duro, batería.

- Asignación de la ponderación, en la tabla 3 se observa un ejemplo con algunos recursos:

Tabla 3. Ponderación criterios, ejemplo

\begin{tabular}{|c|c|}
\hline Criterio & Ponderación \\
\hline Ram & 4 \\
\hline Procesador & 5 \\
\hline Disco Duro & 3 \\
\hline Batería & 5 \\
\hline
\end{tabular}


- Indicar en cuanto satisface la ponderación anterior a cada alternativa, (tabla 4).

Tabla 4. Satisfacción criterios

\begin{tabular}{|c|c|c|c|}
\hline Criterio & Ponderación $\left(\boldsymbol{w}_{\boldsymbol{i}}\right)$ & $\mathbf{S i}\left(\boldsymbol{r}_{\boldsymbol{i 1}}\right)$ & No $\left(\boldsymbol{r}_{\boldsymbol{i} \mathbf{}}\right)$ \\
\hline Ram & 4 & 7 & 7 \\
\hline Procesador & 5 & 6 & 8 \\
\hline Disco Duro & 3 & 5 & 6 \\
\hline Batería & 5 & 6 & 3 \\
\hline
\end{tabular}

- Calcular la ponderación para cada alternativa (tabla 5).

Tabla 5. Cálculo del Score para cada una de las alternativas

\begin{tabular}{|c|c|c|c|c|c|}
\hline $\begin{array}{c}\text { Criteri } \\
0\end{array}$ & $\begin{array}{l}\text { Pondera } \\
\text { ción }\left(w_{i}\right)\end{array}$ & $\begin{array}{c}\mathbf{S i} \\
( \\
r_{i 1} \\
)\end{array}$ & $\begin{array}{l}\text { No } \\
( \\
\left.r_{i 2}\right)\end{array}$ & $\begin{array}{c}\text { Pondera } \\
\text { ción Si } \\
w_{i} * r_{i 1}\end{array}$ & $\begin{array}{c}\text { Pondera } \\
\text { ción No } \\
w_{i} * r_{i 2}\end{array}$ \\
\hline Ram & 4 & 7 & 7 & 28 & 28 \\
\hline $\begin{array}{c}\text { Procesa } \\
\text { dor }\end{array}$ & 5 & 6 & 8 & 30 & 40 \\
\hline $\begin{array}{l}\text { Disco } \\
\text { Duro }\end{array}$ & 3 & 5 & 6 & 15 & 18 \\
\hline Batería & 5 & 6 & 3 & 30 & 15 \\
\hline $\begin{array}{c}\text { Score } \\
\left(\boldsymbol{S}_{\boldsymbol{j}}\right)\end{array}$ & & & & 103 & 101 \\
\hline
\end{tabular}

Para la decisión final, se decidió incluir un indicador de egoísmo (ie) el cual influye en el comportamiento del nodo haciéndolo más egoísta o altruista, esto se hace mediante una distribución Beta que al azar simula el estado de ánimo del nodo y como se observa en la figura 4, hace que el nodo tenga más posibilidades de ser altruista, esto por tratarse de un ambiente de amigos o colegas de trabajo (Fitzek \& Katz, 2014); en donde todos tienden a colaborar.

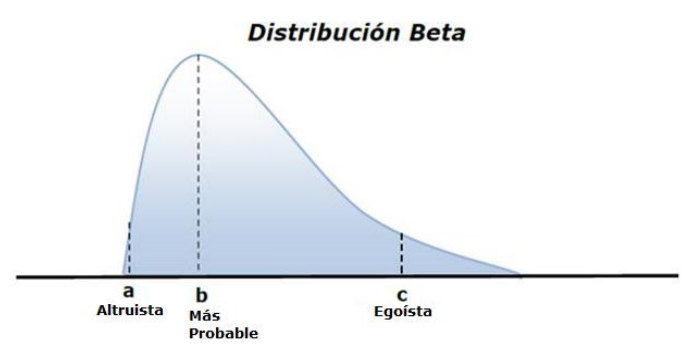

Figura 4. Función de activación Sigmoidal.

Finalmente para incluir este índice y los pesos de las decisiones se introducen estos valores en una función de activación sigmoidal que tiene la forma de la figura 5 y dependiendo de la salida de la función, si es 1 , el nodo ingresa, si es 0 , el nodo no lo hace.

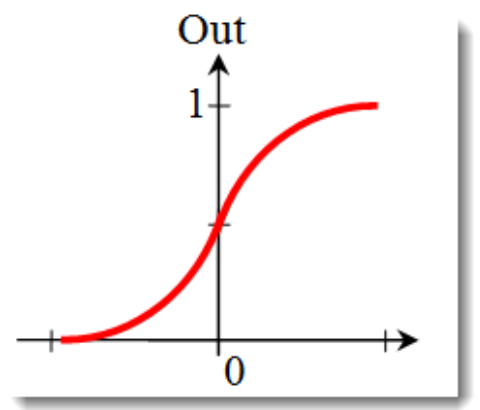

Figura 5. Función de activación Sigmoidal.

La función de la ecuación Sigmoidal (4) es la siguiente:

$$
\text { Out }=\frac{1}{1+e^{(p s-p n * i e)}}
$$


Donde:

$p n$ : Ponderación No

ps: Ponderación Si

ie: Índice de Egoísmo-Altruismo

Con un ie de 0.3, el resultado de Out es 1, por lo que el nodo decide entonces ingresar al sistema distribuido.

\subsection{Nodo con Otra red ya Constituida.}

El segundo caso es cuando un nodo se encuentra a una red Ad Hoc ya conformada; en este caso el nodo se comportaría como una persona al intentar ingresar a una organización o institución; y el sistema distribuido como el grupo que debe aceptar o no a un potencial integrante. $\mathrm{La}$ red debe tener en cuenta el comportamiento del nodo a la hora de dejarlo ingresar, ya que éste se puede comportar de una manera egoísta y no aportar recursos suficientes, esto debe ser claro ya que este sistema debe velar por los recursos de cada uno de sus integrantes y compartirlos de una manera justa (Pitt et al., 2015); se seguirían los pasos observados en la figura 6:

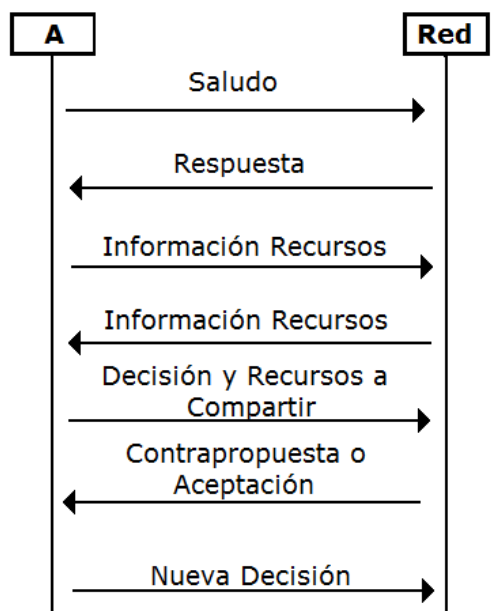

Figura 6. Proceso de formación de la red Ad Hoc, nodo con red Ad Hoc.

El nodo realiza un saludo a la red y ella le envía una respuesta, inmediatamente el nodo envía la información de sus recursos disponibles y totales, de igual manera la red le responde con la información de sus recursos. Luego el nodo toma una decisión inicial de ingresar a la red, por lo cual, transmite una clase de propuesta tentativa de cuáles son los recursos con los cuales va a aportar al sistema, esta elección la hace teniendo en cuenta su estado interno, es decir, su cantidad de batería, recursos disponibles y hasta su deseo de mejorar su reputación en la red. Luego, el sistema distribuido, con base en la teoría social de negociación, toma una decisión, si los recursos que está ofreciendo el nodo son suficientes, la red le envía un mensaje de aceptación para que haga parte de la red, de lo contrario, envía una contrapropuesta, es decir, le indica al nodo que colabore con una cantidad adicional de recursos para que pueda ser aceptado ya que no son suficientes los que propone, si el nodo está de acuerdo con esa contrapropuesta, envía un mensaje de aceptación e ingresa a la red, de lo contrario, el nodo no se incorpora a la red y seguiría escaneando el espectro en busca de otras redes en donde pueda ser aceptado.

Para el método de negociación cooperativa que realiza la red para decidir si acepta el nodo y la posterior negociación que se realiza, se analizan los métodos de negociación cooperativa.

\subsubsection{Principio de Concesiones Alternativas de Zeuthen.}

El método propuesto por Zeuthen, es un procedimiento de negociación en donde las partes hacen ofertas alternativas. Para la formalización, se puede suponer lo siguiente. Digamos que existen el jugador $1 \mathrm{y}$ el jugador 2 . El jugador 1, propone $x=\left(x_{1}, x_{2}\right)$ y el agente 2 , propone $y=\left(y_{1}, y_{2}\right)$, donde el primer componente se refiere a la propuesta para el jugador 1. Se propone un caso de no acuerdo, en donde, en términos de utilidades, el jugador 1 recibiría $u_{1}\left(x_{01}\right)$ y el jugador $2 u_{2}\left(x_{02}\right)$, donde $x_{o}=\left(x_{01}, x_{02}\right)$ sería la situación de conflicto.

Se asume que:

$$
\begin{gathered}
u_{1}\left(x_{01}\right)<u_{1}\left(y_{1}\right)<u_{1}\left(x_{1}\right) \mathrm{y} \\
u_{2}\left(x_{02}\right)<u_{2}\left(x_{2}\right)<u_{2}\left(y_{2}\right)
\end{gathered}
$$

En la ecuación (5) se resume que el jugador prefiere su propuesta a la del otro jugador, pero prefiere estas dos situaciones a la situación de conflicto.

El procedimiento pues, para las situaciones a las que se enfrentan ambos jugadores, sería el siguiente. Digamos que $x$ es la última propuesta del jugador 1 y $y$ la última propuesta del jugador 2. Suponiendo que ambos jugadores son maximizadores bayesianos de la utilidad, entonces se puede suponer que $p_{12}$ es una 
probabilidad subjetiva que el jugador 1 asigna a que el jugador 2 se apegue a su última oferta, es decir $y$. Y $\left(1-p_{12}\right)$ la probabilidad subjetiva de que el jugador 2 aceptará la oferta hecha por el jugador 1 .

Si el jugador 1 acepta la última oferta del oponente, el jugador 1 recibirá $u_{1}\left(y_{1}\right)$. Si el jugador 1 se apega a su última oferta personal, el jugador 1 obtendrá $u_{1}\left(x_{1}\right)>u_{1}\left(y_{1}\right)$ con una probabilidad de $\left(1-p_{12}\right)$, pero también podría obtener la utilidad mínima $u_{1}\left(x_{01}\right)<u_{1}\left(y_{1}\right)$, con una probabilidad $p_{12}$. De manera que si se quiere maximizar la ganancia, el jugador 1 , se apegaría a su última oferta $x$, si y solo si se cumple lo indicado en la ecuación (6):

$$
\left(1-p_{12}\right) \cdot u_{1}\left(x_{1}\right)+p_{12} \cdot u_{1}\left(x_{01}\right) \geq u_{1}\left(y_{1}\right)
$$

Lo que es igual a (7):

$$
p_{12} \leq \frac{u_{1}\left(x_{1}\right)-u_{1}\left(y_{1}\right)}{u_{1}\left(x_{1}\right)-u_{1}\left(x_{01}\right)}
$$

Esta relación se llama límite de riesgo $r_{1}$, es el riesgo máximo que el jugador 1 , está dispuesto a enfrentar para establecer un arreglo de acuerdo a su propia propuesta más que en los términos que le ofrece su oponente.

Para ello, el autor propone las siguientes reglas de decisión para saber cuál de los dos jugadores realizará la primera concesión, es decir, quién va a ceder un poco de su utilidad para poder lograr un acuerdo entre las dos partes.

a) Si $r_{1}>r 2$, entonces el jugador 2 tiene que hacer la siguiente concesión.

b) Si $r_{1}<r 2$, entonces el jugador 1 tiene que hacer la siguiente concesión.

c) Si $r_{1}=r 2$, entonces ambos jugadores tienen que ceder un poco.

Digamos que el jugador 1 propone $x=\left(x_{1}, x_{2}\right)$, mientras que el jugador 2 propone $y=\left(y_{1}, y_{2}\right)$, entonces se cumple lo indicado en la ecuación (8):

$$
\begin{gathered}
r_{1}=\frac{u_{1}\left(x_{1}\right)-u_{1}\left(y_{1}\right)}{u_{1}\left(x_{1}\right)-u_{1}\left(x_{01}\right)} \leq \frac{u_{2}\left(y_{2}\right)-u_{2}\left(x_{2}\right)}{u_{2}\left(y_{2}\right)-u_{2}\left(x_{02}\right)} \\
=r_{2}
\end{gathered}
$$

O lo que es lo mismo (9),

$$
\begin{gathered}
\left(u_{1}\left(x_{1}\right)-u_{1}\left(x_{01}\right)\right) \cdot\left(u_{2}\left(x_{2}\right)-u_{2}\left(x_{02}\right)\right) \leq \\
\left(u_{1}\left(y_{1}\right)-u_{1}\left(x_{01}\right)\right) \cdot\left(u_{2}\left(y_{2}\right)-u_{2}\left(x_{02}\right)\right)
\end{gathered}
$$

De acuerdo a las reglas de Zeuthen, $r_{1}$ tendría que hacer la concesión, si se especifica que la nueva concesión es $x^{\prime}=\left(x_{1}^{\prime}, x^{\prime}{ }_{2}\right)$, entonces se tiene la inecuación (10):

$$
\begin{gathered}
r_{1}^{\prime}=\frac{u_{1}\left(x_{1}^{\prime}\right)-u_{1}\left(y_{1}\right)}{u_{1}\left(x_{1}^{\prime}\right)-u_{1}\left(x_{01}\right)} \leq \frac{u_{2}\left(y_{2}\right)-u_{2}\left(x_{2}^{\prime}\right)}{u_{2}\left(y_{2}\right)-u_{2}\left(x_{02}\right)} \\
=r^{\prime}{ }_{2}
\end{gathered}
$$

O su equivalente (11),

$\left(u_{1}\left(y_{1}\right)-u_{1}\left(x_{01}\right)\right) \cdot\left(u_{2}\left(y_{2}\right)-u_{2}\left(x_{02}\right)\right) \leq$ $\left(u_{1}\left(x_{1}^{\prime}\right)-u_{1}\left(x_{01}\right)\right) \cdot\left(u_{2}\left(x_{2}^{\prime}\right)-u_{2}\left(x_{02}\right)\right)$

Como se puede observar, en la primera productoria de Nash del jugador 1, es menor a la productoria del jugador 2, por lo cual el jugador 1 realiza la primera concesión, luego, en la siguiente ronda, la productoria de Nash del jugador 2, es menor que la del jugador 1, por lo cual, éste debe hacer la siguiente oferta. En cada ronda, se producirá una productoria de Nash más alta, así sucesivamente hasta que en algún momento, algún jugador haga una oferta con el valor más alto posible del producto de Nash. Este punto sería el que aceptarían los dos jugadores y en el cual se obtendría un acuerdo.

\section{RESULTADOS}

Para validar los resultados se hizo la simulación del experimento mediante Python, probando con cinco familias de dispositivos: computadores, celulares, tablets, equipos embebidos como Raspberries Pi y Beagle Backbone, con los recursos que en promedio tienen cada uno de ellos. Para realizar una normalización de los parámetros y tomando como base que el proyecto de tesis se va a realizar sobre equipos Raspberry Pi, se hizo una traducción a una escala numérica de los recursos de cada uno de los dispositivos, y se hizo el procedimiento tal y como se mostró anteriormente en un escenario en el cual cada nodo quería realizar el proceso de unión al sistema distribuido. 
En un ambiente de pruebas común con una red de más de 5 nodos (teniendo en cuenta que se utilizaron dispositivos como Raspberry o computadores), se realizaron las mediciones de los pesos de los recursos y se colocó como parámetro estándar un peso o Score de 150 para la red.

Luego se investigó sobre cada una de las familias de dispositivos y se hizo un promedio colocando como base las ponderaciones realizadas anteriormente y para cada uno de ellos se obtuvieron los valores de la Tabla No. 6; además se añadió un índice de altruismoegoísmo, es decir, un parámetro que influenciaba al nodo a comportarse de cierta manera y aumentar las posibilidades de ingreso a la red, esto, teniendo en cuenta el comportamiento del individuo en la comunidad y su disposición a colaborar de manera altruista como un incentivo para mejorar su reputación o estatus en la sociedad y de igual forma a ser tenido en cuenta por la red a la hora de la asignación de recursos cuando sea requerido por el dispositivo.

Con estos datos se procedió a la simulación en donde se obtuvieron los resultados de la Tabla 6.

Tabla 6. Porcentaje de ingreso de dispositivos a la red

\begin{tabular}{|c|c|}
\hline Dispositivo & $\begin{array}{c}\text { Porcentaje } \\
\text { ingreso }\end{array}$ \\
\hline Raspberry Pi & $93,7 \%$ \\
\hline Beagle Backbone & $91,11 \%$ \\
\hline Celular & $97,7 \%$ \\
\hline Computador & $80,2 \%$ \\
\hline Tablet & $75,5 \%$ \\
\hline
\end{tabular}

Los anteriores resultados se explican por el hecho de que los nodos que menos recursos tienen, ven en la red la oportunidad de acceder a más recursos y poder aumentar sus posibilidades de satisfacción de las necesidades, mientras que los dispositivos que más tienen recursos como los computadores, ya cuentan con una gran cantidad de recursos y uno de sus principales incentivos para el ingreso sería el ganar reputación en la misma.

\section{CONCLUSIONES}

La toma de decisiones multicriterio discreta por su definición es la mejor manera de abordar este problema, ya que considera un conjunto de alternativas discreto y el método Scoring en específico, tiene el componente de permitir establecer una función de utilidad de manera que el decisor pueda indicar cuales son los criterios que más prefiere a la hora de tomar la decisión.

Los métodos multicriterios como Electre documentado en (Deb, 2001), utiliza los conceptos de concordancia, discordancia y valores de umbral para establecer una relación de preferencia entre las alternativas, luego crea un grafo que refleje esa preferencia, pero puede darse el caso que el grafo elimine algunas de las opciones de tal manera que los valores de preferencia tienen que ser prestablecidos. El método AHP (Moreno, 2002) es muy similar al método Scoring, en donde existe también la posibilidad de añadir aspectos intangibles en la toma de decisiones como el subjetivismo y la incertidumbre, el método requiere que se cree una matriz de comparación que compare las alternativas por pares, entre más alternativas exista, el método se vuelve un poco más complejo.

Para la segunda parte de la toma de decisiones en donde el sistema distribuido acepta al nodo basándose en la cantidad de recursos que ofrece, el método de concesiones alternativas de Zeuthen es un buen enfoque ya que permite de una manera sencilla hacer una negociación maximizando la utilidad de los autores involucrados.

Los dispositivos con capacidad de recursos más baja como celulares y Raspberry Pi ven una oportunidad de aumentar las posibilidades de acceso a recursos al ingresar a una red, mientras que los dispositivos con más recursos como computadores, ven la posibilidad de aumentar su reputación siendo altruistas a la hora de compartir recursos.

En este artículo se expuso la teoría sobre diferentes clases de métodos de toma de decisiones enmarcado en el proyecto TLÖN de la Universidad Nacional que busca ser un primer paso hacia la adición de un paradigma social-inspirado en sistemas distribuidos, se vieron definiciones relacionadas a los aspectos más importantes sobre toma de decisiones. 


\section{RECOMENDACIONES}

El número de negociaciones que se realiza entre el nodo y el sistema distribuido en el momento en el cual negocian la cantidad de recursos que se van a entregar a la red debe reducirse al mínimo posible ya que estas transacciones aumentarían los costos de procesamiento y retardarían el ingreso a la red.

Por ser un primer paso hacia la Social-inspiración, este proyecto encaja muy bien en el que se está llevando a cabo en el grupo de investigación TLÖN de la Universidad Nacional de tal manera que los trabajos futuros deben consistir en la integración de lo realizado en el sistema distribuido.

\section{AGRADECIMIENTOS}

Agradezco enormemente a mis compañeros y a los docentes del grupo de investigación TLÖN de la Universidad Nacional de Colombia ya que entre todos estamos realizando un gran proyecto y es gratificante saber que cuento con grandes profesionales que siempre están ahí para apoyarme en lo que necesite.

\section{REFERENCIAS}

Crawford, V.P., 1980. A Note on the Zeuthen-Harsanyi theory of bargaining. Journal of Conflict Resolution, 24(3), pp.525-535. https://doi.org/10.1177/002200278002400307

Fitzek, F. \& Katz, M., 2014. MOBILE CLOUDS: Exploiting Distributed Resources in Wireless, Mobile and Social Networks L. John Wiley \& Sons, ed., New Delhi, India. ISBN: 978-0-470-97389-9

Deb, K., 2001. Multi-objective Optimization Using Evolutionary Algorithms. Vol. 16. Califonia: John Wiley \& Sons.

Gaertner, W., 2009. A Primer in Social Choice Theory Revised., Oxford: Oxford University Press. ISBN: 9780199565306

Kalai, E., 1977. Proportional Solutions to Bargaining Situations: Interpersonal Utility Comparisons. Econometrica, 45(7), pp.1623-1630. DOI: 10.2307/1913954

Loo, J., Lloret, J. \& Hamilton, J., 2012. Mobile Ad Hoc Networks: Current Status and Future Trends, Taylor \& Francis Group, LLC. ISBN 9781439856505 - CAT\# K12654

Maldonado, C.E. \& Gomez, N.A., 2011. Sistemas Bio- inspirados: Un Marco Teórico para la Ingeniería de Sistemas Complejos, Bogotá. ISSN: 0124-8219

Moreno Jiménez, J., 2002. El Proceso Análitico Jerárquico (Ahp). Fundamentos, Metodología y Aplicaciones, En Recta 1, p. 21-53.

Ozdaglar, A., 2010. Existence of a Nash Equilibrium. MIT OPEN COURSE WARE, p.28. https://ocw.mit.edu/courses/electrical-engineering-andcomputer-science/6-254-game-theory-with-engineeringapplications-spring-2010/lecture-

notes/MIT6_254S10_lec05.pdf Consultado Noviembre 15 de 2017.

Pitt, J., Busquets, D., \& Riveret, R. (2015). The pursuit of computational justice in open systems. AI \& society, 30(3), 359-378. doi: $10.1007 / \mathrm{s} 00146-013-0531-6$

Rawls, J. (2012). Teoría de la justicia. Fondo de cultura económica.

Saaty, T. L. (1990). How to make a decision: the analytic hierarchy process. European journal of operational research, 48(1), 9-26. https://doi.org/10.1016/03772217(90)90057-I

Stiefken Arboleda, J.P., 2008. Altruismo y solidaridad en el Estado de Bienestar. Universidad Autónoma de Barcelona. https://ddd.uab.cat/pub/trerecpro/2008/hdl_2072_9974/Treba 11_de_recerca.pdf. Consultado Noviembre 17 de 2017.

Toh, C.K. et al., 2010. The controversy of Selfish nodes in ad hoc networks. Advanced Communication Technology (ICACT), 2010 The 12th International Conference on, 2.

Vitoriano, B., 2007. Teoría de la decisión: decisión con incertidumbre, decisión multicriterio y teoría de juegos. Universidad Complutense de Madrid, p.107. $\mathrm{http} / / /$ moodle2.unid.edu.mx/dts_cursos_mdl/pos/AN/MTD/A M/10/Teoria.pdf. Consultado 7 de Enero 2018.

Zarate Ceballos, H. et al., 2015. Sistema de Telecomunicaciones Social-Inspirado mediante Comunidades de Agentes. In N. Becerra Correa, ed. Congreso Internacional de Computación Colombia-México. Cartagena: FABBERCOR.ONG, pp. 56-63. 